Doi: HTTPS://DOI.ORG/10.23910/IJBSM/2018.9.2.3C0157

\title{
Effect of Upland Rice Varieties on Relative Composition of Weeds in Jharkhand
}

\author{
Sheela Barla* and R. R. Upasani
}

Dept. of Agronomy, Birsa Agricultural University, Ranchi, Jharkhand (834 006), India

\section{Corresponding Author}

Sheela Barla

e-mail: sheela.barla123@gmail.com

\author{
Article History \\ Article ID: 3 C0157 \\ Received in $17^{\text {th }}$ September, 2017 \\ Received in revised form $28^{\text {th }}$ December, 2017 \\ Accepted in final form 24 ${ }^{\text {th }}$ March, 2018
}

\begin{abstract}
Weed management in upland rice production is a major constraint in reducing rice yield in Jharkhand state. Studies were conducted during the wet cropping season of 2011 and 2012 at Zonal Research Station, East Singhbhum under upland ecology to assess and identify crop parameters responsible for competitiveness of rice varieties. Total thirteen upland varieties including ten improved and three traditional varieties were tested under weedy and weed free conditions. The major weeds found in the experimental plot were Bulbostilis barbata, Ludwigia parviflora, Cyperus rotundus, Alternanthera sessilis and Cynodon dactylon The results indicated that rice varieties differed in their competitiveness against weeds. Bulbostilis barbata was found as a major weed at 25, 50 and 75 DAS with average relative composition of $44.39,41.37$ and $46.82 \%$ in weedy condition, while $32.15,30.46$ and $22.92 \%$ under two hand weeding at 20 and 40 days after sowing. The varieties Vandana and Anjali were found to be most competitive and productive, whilst among local varieties, Tanrbhojna though competitive in suppressing weeds but was not much productive. Maximum yield loss of $29.86 \%$ occurred when no weeding was performed irrespective of rice varieties. Among different improved rive varieties BVD-110, Vandana and Anjali recorded yield loss to the tune of $5.24,7.01$ and $8.6 \%$ respectively. However, traditional varieties Asanleva, Balibhojna and Tanrbhojnarecorded yield loss to the extent of $4.59,4.83$ and $6.02 \%$ indicating weed suppressing ability compared to others.
\end{abstract}

Keywords: Varieties, competitiveness, upland rice

\section{Introduction}

Dry direct seeding of rice is a common practice among farmers in West Singhbhum and Saraikela -Kharsawan Districts of Jharkhand due to uncertainty of monsoon, water crisis as well as scarcity of labour. Majority of the farmers are marginal having more acreage under upland than medium or low land. Growing rice in upland condition is a great challenge to the marginal farmers as weeds are among the most important biological constraints for successful production of direct seeded rice (DSR). DSR production system is subject to greater weed pressure than conventional transplant method of production systems, in which weeds are suppressed by puddling as well as transplanting of 20-30 days old well developed seedlings having an edge over germinating weeds. The problem of weed competition in upland rice is of great economic importance as it may cause $50-91 \%$ reduction in grain yield. The initial forty days after rice seeding have been found to be critical with respect to crop weed competition (Singh et al., 2012). Many options exists for weed control in DSR, perhaps the most common being the use of herbicides, but higher cost of herbicide, effect on environment, incorrect use of herbicides may bring about other environmental problems (Labrada, 2003), besides evaluation of herbicide resistant weed, unavailability of herbicide, lack of requisite knowledge and skill to use the herbicide correctly are the major concerned that needs to be taken care of. Hence, Alternative weed management technologies are therefore much needed. Variation among genotypes in their ability to compete with weeds has been documented for many crops, including rice (Zhao et al., 2006). Although some studies exist on the differences in competitiveness (Fischer et al. 2001; Anwar et al., 2010), only a limited number of cultivars have been evaluated so far. Weed competitive rice cultivars particularly traditional varieties, which have higher adaptability and food habitparticularly used as puffed rice needed for sustainable upland rice production.The identification and development of competitive rice varieties may be effective in weed suppression and provide a tool for integrated weed management (Fischer et al., 2001; Caton et al., 2003). Contrary to other weed control methods, improved varieties have proven well for ease of adoption. Some of the rice varieties grow quickly and produce early canopy resulting in shading and thus suppress weed growth. Keeping these in view the experiment was formulated in the upland ecology to assess the competitiveness of different rice varieties and identify plant 
parameters responsible for their competitiveness. Jharkhand is endowed with a number of local varieties of rice grown in different pockets of Jharkhand which have been found to smother weeds thus have competitive edges. However, screening of these varieties is yet to be explored in context to efficient weed management as well as their productivity under upland un irrigated condition.

\section{Materials and Methods}

A field experiment was carried out during rainy seasons of 2011 and 2012 at zonal research station, East Singhbhum, Jharkhand $\left(22^{\circ} 41^{\prime} \mathrm{N}\right.$ latitude and $86^{\circ} 23^{\prime} \mathrm{E}$ longitude with an altitude of $124 \mathrm{~m}$ above mean sea level) in the upland ecology to assess the competitiveness of different rice varieties and to identify plant parameters responsible for their competitiveness. The soil of experimental plot was silt loam in texture with $\mathrm{pH}$ 6.2. The soil was low in available $\mathrm{N}(250.45 \mathrm{~kg}$ ha-1), medium in $\mathrm{P}_{2} \mathrm{O}_{5}\left(16.62 \mathrm{~kg} \mathrm{ha}^{-1}\right)$ and $\mathrm{K}_{2} \mathrm{O}$ (135.19 kg ha-1). The experiment was laid out in split plot design. Thirteen rice varieties were tested under weed free i.e farmers practice, two hand weeding at 20 and 40 days after sowing (DAS) and weedy check. The treatments were replicated thrice. Line sowing was done at a spacing of $20 \mathrm{~cm}$. Recommended dose of fertilizer 60:30:20 kg N, $\mathrm{P}_{2} \mathrm{O}_{5}$ and $\mathrm{K}_{2} \mathrm{O}$ ha-1 respectively, was applied through urea, single super phosphate and muriate of potash.
Half of the nitrogen, full dose of phosphorus and potassium were applied before sowing. Remaining half of nitrogen was applied in two equal splits at tillering and panicle initiation stage of crop. The yield attributing parameters and yield of the crop were recorded after physiological maturity. Weeds were counted species-wise and differentiated into categories of sedges, grass, and broadleaf weeds. Relative composition of weeds (\%) of an individual weed species was calculated by the formula as suggested by Shetty and Rao (1979).

$\begin{aligned} & \text { Relative composition } \\ & \text { of a species }(\%)=\end{aligned} \quad \frac{\text { No. on individual species }}{\text { Total no.of all weeds }} \times 100(\%)$

The relative yield loss (YL) of the crop challenged by weed competition under field conditions was estimated using equation, $\mathrm{YL}(\%)=1-(\mathrm{YCW} / \mathrm{YCM}) \times 100$ where, $Y C W$ and $Y C M$ are crop yields in competition with weeds and in weed-free conditions, respectively (Harding and Jalloh, 2013).

\section{Results and Discussion}

\subsection{Effect on relative composition of weed species}

Relative composition of weed species differed under different rice varieties.Weed Bulbostilis barbata was found as a major weed at 25, 50 and 75 DAS (Table 1, 2 and 3) with average relative composition of $44.39,41.37$ and $46.82 \%$ in weedy condition while $32.15,30.46$ and $22.92 \%$ under

Table 1: Relative composition of weeds (\%) at 25 DAS as affected by different varieties

\begin{tabular}{|c|c|c|c|c|c|c|c|c|c|c|c|c|}
\hline \multirow[t]{3}{*}{ Rice varieties } & \multicolumn{12}{|c|}{ Weed species } \\
\hline & \multicolumn{2}{|c|}{$\begin{array}{l}\text { Cyperus } \\
\text { rotundus }\end{array}$} & \multicolumn{2}{|c|}{$\begin{array}{l}\text { Cynodon } \\
\text { dactylon }\end{array}$} & \multicolumn{2}{|c|}{$\begin{array}{c}\text { Bulbostilis } \\
\text { burbata }\end{array}$} & \multicolumn{2}{|c|}{$\begin{array}{l}\text { Ludwigia } \\
\text { parviflora }\end{array}$} & \multicolumn{2}{|c|}{ Other NL } & \multicolumn{2}{|c|}{ Other BL } \\
\hline & Weedy & $\begin{array}{l}\text { Weed } \\
\text { free }\end{array}$ & Weedy & $\begin{array}{l}\text { Weed } \\
\text { free }\end{array}$ & Weedy & $\begin{array}{l}\text { Weed } \\
\text { free }\end{array}$ & Weedy & $\begin{array}{l}\text { Weed } \\
\text { free }\end{array}$ & Weedy & $\begin{array}{l}\text { Weed } \\
\text { free }\end{array}$ & Weedy & $\begin{array}{c}\text { Weed } \\
\text { free }\end{array}$ \\
\hline Vandana & 17.51 & 7.42 & 10.47 & 3.96 & 53.32 & 34.64 & 23.74 & 21.77 & 10.32 & 8.86 & 1.65 & 1.25 \\
\hline BVD 109 & 13.85 & 12.49 & 10.33 & 8.22 & 59.47 & 42.34 & 28.94 & 10.52 & 4.40 & 3.54 & 3.62 & 0.87 \\
\hline BVD 110 & 17.09 & 15.23 & 10.19 & 2.47 & 38.07 & 37.78 & 30.74 & 24.85 & 5.02 & 4.67 & 6.96 & 6.92 \\
\hline Anjali & 23.22 & 14.19 & 10.63 & 6.39 & 43.88 & 26.10 & 28.27 & 23.52 & 11.02 & 4.57 & 4.99 & 3.21 \\
\hline Kalinga 3 & 16.45 & 15.87 & 7.66 & 4.59 & 42.11 & 34.75 & 32.53 & 27.45 & 5.38 & 3.61 & 6.60 & 2.72 \\
\hline $\begin{array}{l}\text { Brown gora } \\
102\end{array}$ & 18.88 & 18.84 & 9.65 & 7.79 & 40.20 & 33.48 & 23.88 & 20.88 & 7.11 & 1.47 & 14.07 & 3.33 \\
\hline Annada & 17.42 & 12.48 & 7.84 & 4.65 & 41.48 & 41.45 & 27.42 & 21.78 & 11.28 & 5.84 & 5.67 & 2.70 \\
\hline Narendra 97 & 20.89 & 15.84 & 8.65 & 8.42 & 43.55 & 33.75 & 24.83 & 24.07 & 4.02 & 2.71 & 9.40 & 3.85 \\
\hline BirsaDhan 101 & 20.48 & 13.63 & 7.92 & 5.44 & 48.26 & 34.97 & 26.54 & 21.30 & 11.09 & 5.02 & 4.24 & 1.12 \\
\hline BirsaDhan 108 & 27.20 & 12.61 & 10.78 & 5.42 & 43.50 & 19.07 & 32.58 & 30.30 & 4.80 & 4.03 & 8.62 & 1.08 \\
\hline Tanrbhojna & 24.68 & 15.18 & 7.29 & 4.58 & 38.31 & 14.65 & 47.00 & 35.10 & 3.70 & 2.48 & 3.91 & 3.13 \\
\hline Asanleva & 12.91 & 12.90 & 6.84 & 4.79 & 49.07 & 46.26 & 30.56 & 21.39 & 3.97 & 3.54 & 4.43 & 1.94 \\
\hline Balibhojna & 11.67 & 9.70 & 10.46 & 6.04 & 47.06 & 37.87 & 42.60 & 23.02 & 6.43 & 1.60 & 3.33 & 0.22 \\
\hline $\begin{array}{l}\text { Mean Relative } \\
\text { weed compo- } \\
\text { sition (\%) }\end{array}$ & 19.45 & 13.25 & 9.00 & 5.82 & 44.39 & 32.15 & 31.68 & 24.35 & 6.64 & 3.98 & 6.05 & 2.68 \\
\hline
\end{tabular}




\begin{tabular}{|c|c|c|c|c|c|c|c|c|c|c|c|c|}
\hline \multirow[t]{3}{*}{ Rice varieties } & \multicolumn{12}{|c|}{ Weed species } \\
\hline & \multicolumn{2}{|c|}{$\begin{array}{l}\text { Cyperus } \\
\text { rotundus }\end{array}$} & \multicolumn{2}{|c|}{$\begin{array}{l}\text { Cynodon } \\
\text { dactylon }\end{array}$} & \multicolumn{2}{|c|}{$\begin{array}{l}\text { Bulbostilis } \\
\text { burbata }\end{array}$} & \multicolumn{2}{|c|}{$\begin{array}{l}\text { Ludwigia } \\
\text { parviflora }\end{array}$} & \multicolumn{2}{|c|}{ Other NL } & \multicolumn{2}{|c|}{ Other BL } \\
\hline & Weedy & $\begin{array}{c}\text { Weed } \\
\text { free }\end{array}$ & Weedy & $\begin{array}{l}\text { Weed } \\
\text { free }\end{array}$ & Weedy & $\begin{array}{c}\text { Weed } \\
\text { free }\end{array}$ & Weedy & $\begin{array}{c}\text { Weed } \\
\text { free }\end{array}$ & Weedy & $\begin{array}{l}\text { Weed } \\
\text { free }\end{array}$ & Weedy & $\begin{array}{l}\text { Weed } \\
\text { free }\end{array}$ \\
\hline Vandana & 23.29 & 20.24 & 8.15 & 3.99 & 43.30 & 32.48 & 16.92 & 14.00 & 14.03 & 8.11 & 8.18 & 7.30 \\
\hline BVD 109 & 21.50 & 8.59 & 10.03 & 3.00 & 50.70 & 29.53 & 19.12 & 18.36 & 8.89 & 5.89 & 12.70 & 11.69 \\
\hline BVD 110 & 14.92 & 13.06 & 7.45 & 6.57 & 49.05 & 46.13 & 13.60 & 8.71 & 10.51 & 7.50 & 15.11 & 7.39 \\
\hline Anjali & 28.88 & 13.79 & 10.37 & 4.88 & 46.52 & 40.55 & 10.64 & 8.13 & 7.09 & 5.57 & 12.00 & 11.60 \\
\hline Kalinga 3 & 8.65 & 7.91 & 17.30 & 4.62 & 53.18 & 36.22 & 18.92 & 18.88 & 5.20 & 4.25 & 14.49 & 10.37 \\
\hline Brown gora 102 & 17.33 & 10.47 & 5.03 & 2.65 & 51.14 & 37.07 & 32.94 & 10.05 & 7.07 & 6.08 & 10.79 & 9.37 \\
\hline Annada & 17.37 & 12.13 & 8.27 & 5.79 & 39.47 & 31.45 & 18.45 & 9.13 & 9.70 & 7.15 & 31.87 & 9.23 \\
\hline Narendra 97 & 30.04 & 9.84 & 11.41 & 4.56 & 31.32 & 25.15 & 33.34 & 10.84 & 12.44 & 6.41 & 14.54 & 10.12 \\
\hline BirsaDhan 101 & 25.30 & 13.59 & 12.67 & 3.92 & 40.17 & 17.24 & 18.56 & 16.07 & 8.47 & 7.11 & 19.12 & 17.77 \\
\hline BirsaDhan 108 & 17.41 & 10.90 & 10.10 & 6.01 & 39.92 & 20.32 & 32.56 & 19.16 & 8.35 & 7.91 & 18.20 & 9.14 \\
\hline Tanrbhojna & 23.73 & 13.26 & 7.09 & 6.65 & 29.77 & 27.91 & 19.85 & 12.54 & 10.22 & 5.30 & 23.43 & 20.26 \\
\hline Asanleva & 24.62 & 14.04 & 7.40 & 6.83 & 41.22 & 31.12 & 16.36 & 12.79 & 8.40 & 7.86 & 23.79 & 5.59 \\
\hline Balibhojna & 16.82 & 11.56 & 6.31 & 5.86 & 39.66 & 28.37 & 22.06 & 21.03 & 9.60 & 9.17 & 17.27 & 12.30 \\
\hline $\begin{array}{l}\text { Mean relative weed } \\
\text { composition (\%) }\end{array}$ & 20.39 & 12.50 & 10.07 & 5.08 & 41.37 & 30.46 & 20.63 & 13.98 & 9.08 & 6.67 & 17.76 & 12.00 \\
\hline
\end{tabular}

\begin{tabular}{|c|c|c|c|c|c|c|c|c|c|c|c|c|c|c|}
\hline \multirow[t]{3}{*}{ Rice varieties } & \multicolumn{14}{|c|}{ Weed species } \\
\hline & \multicolumn{2}{|c|}{$\begin{array}{l}\text { Cyperus } \\
\text { rotundus }\end{array}$} & \multicolumn{2}{|c|}{$\begin{array}{l}\text { Cynodon } \\
\text { dactylon }\end{array}$} & \multicolumn{2}{|c|}{$\begin{array}{l}\text { Bulbostilis } \\
\text { burbata }\end{array}$} & \multicolumn{2}{|c|}{$\begin{array}{l}\text { Ludwigia } \\
\text { parviflora }\end{array}$} & \multicolumn{2}{|c|}{$\begin{array}{l}\text { Alternan- } \\
\text { thera sessilis }\end{array}$} & \multicolumn{2}{|c|}{ Other NL } & \multicolumn{2}{|c|}{ Other BL } \\
\hline & W1 & W2 & W1 & W2 & W1 & W2 & W1 & W2 & W1 & W2 & W1 & W2 & W1 & W2 \\
\hline Vandana & 5.59 & 1.14 & 10.94 & 7.16 & 37.63 & 32.37 & 32.84 & 27.29 & 12.20 & 1.92 & 8.71 & 6.44 & 13.40 & 4.29 \\
\hline BVD 109 & 8.12 & 7.31 & 8.37 & 8.09 & 45.36 & 23.18 & 21.32 & 19.57 & 28.50 & 7.59 & 7.92 & 6.62 & 9.34 & 5.92 \\
\hline BVD 110 & 7.06 & 6.69 & 5.78 & 5.42 & 53.22 & 21.25 & 22.91 & 19.05 & 28.24 & 2.82 & 9.83 & 7.69 & 7.43 & 5.12 \\
\hline Anjali & 9.83 & 7.97 & 9.50 & 5.72 & 41.44 & 17.76 & 20.68 & 18.55 & 29.63 & 10.52 & 9.22 & 6.53 & 12.83 & 10.05 \\
\hline Kalinga 3 & 8.89 & 6.49 & 9.80 & 4.57 & 54.09 & 19.16 & 24.73 & 20.65 & 18.96 & 1.14 & 7.46 & 5.29 & 16.24 & 3.68 \\
\hline Brown gora 102 & 13.43 & 5.97 & 5.91 & 5.04 & 51.09 & 33.47 & 26.96 & 20.51 & 16.31 & 1.43 & 7.91 & 5.22 & 4.33 & 3.85 \\
\hline Annada & 6.86 & 5.38 & 8.28 & 4.15 & 50.68 & 25.44 & 27.68 & 15.80 & 12.55 & 8.85 & 11.96 & 7.84 & 12.02 & 11.36 \\
\hline Narendra 97 & 8.06 & 4.86 & 4.48 & 3.32 & 51.95 & 23.83 & 27.54 & 14.72 & 19.26 & 9.62 & 7.81 & 6.62 & 14.57 & 12.58 \\
\hline BirsaDhan 101 & 6.15 & 3.53 & 11.26 & 5.11 & 39.96 & 20.77 & 31.78 & 15.53 & 13.52 & 12.89 & 7.84 & 6.21 & 19.70 & 19.27 \\
\hline BirsaDhan 108 & 7.37 & 4.71 & 8.15 & 2.92 & 43.84 & 17.08 & 31.87 & 19.84 & 21.03 & 8.44 & 9.38 & 8.29 & 13.01 & 12.02 \\
\hline Tanrbhojna & 6.19 & 5.11 & 11.78 & 7.01 & 44.78 & 16.54 & 17.89 & 13.34 & 31.48 & 13.13 & 10.89 & 6.67 & 18.33 & 9.99 \\
\hline Asanleva & 8.77 & 3.53 & 8.98 & 2.58 & 53.45 & 29.81 & 28.62 & 14.09 & 19.20 & 8.38 & 6.87 & 2.97 & 11.75 & 9.39 \\
\hline Balibhojna & 2.68 & 2.20 & 6.93 & 4.35 & 68.13 & 24.34 & 18.16 & 14.29 & 21.81 & 5.35 & 6.10 & 4.44 & 19.97 & 6.59 \\
\hline $\begin{array}{l}\text { Mean relative } \\
\text { weed composi- } \\
\text { tion }(\%)\end{array}$ & 7.65 & 5.05 & 8.64 & 5.05 & 46.82 & 22.92 & 25.12 & 17.53 & 21.67 & 8.66 & 8.90 & 6.45 & 14.55 & 9.56 \\
\hline
\end{tabular}


two hand weeding at 20 and 40 days after sowing, followed by Ludwigia parviflora and Cyperus rotundus. While at 75 DAS Alternanthera sessilis became third major weed after Bulbostilis barbata and Ludwigia parviflora (Table 3).

According to relative weed composition among 13 varieties Vandana recorded minimum population of Cyperus rotundus at 25 and 75 DAS and other broad leaf weeds at 50 and 75 DAS. Variety BVD - 110 recorded minimum of Cynodon dactylon at 25 and 75 DAS. Population of major weed Bulbostilis barbata was minimum with local variety Tanrbhojna throughout crop growth stages. However at 75 DAS Ludwigia parviflora and other narrow leaf weeds were also recorded minimum with this variety. Variety BVD - 109, BVD- 110 and Anjali also recorded reduced population of Ludwigia parviflora. Percentage decrease in weed density of Cyperus rotundus, Cynodon dactylon and Alternanthera sessilis over weedy check is more with variety Vandana. The mean relative weed composition is maximum with Alternanthera sessilis (60.06\%) at 75 DAS (Table 4). Thus, varietal differences had pronounced weed suppression capabilities. Variety Vandana reduce weed biomass more than other varieties irrespective of weeding regime.

\subsection{Effect on rice}

Two hand weeding at 20 and 40 days after sowing recorded enhanced effective tillers, grains panicle ${ }^{-1}, 1000$ grain weight compared to weedy check. The increase was to the tune of $9.44,32.43$ and $4.06 \%$ respectively. Maximum value of yield attributes in two hand weeding at 20 and 40 days after sowing were also reported by Mishra and Singh (2008). Among varieties Vandana similar to Anjali and BVD-109 had more growth and yield attributing characters than other varieties irrespective of the weeding regime. Number of days taken for anthesis and maturity differed among varieties. Un weeded plot recorded delayed anthesis as well as delayed maturity owing to high inter specific competition for resources (Evans et al., 2003).Two hand weeding at 20 and 40 days after sowing affected flowering and maturity. Uncontrolled weed growth delayed flowering and maturity by 4-6 days compared with other varieties in weedy check.

\subsection{Grain yield, loss and economics}

Two hand weeding at 20 and 40 days after sowing recorded $33.62 \%$ higher grain yield ( $2212 \mathrm{~kg} \mathrm{ha}^{-1}$ ) compared to weedy check (Table 5). Jadhav and Pawar, 2013; Kumar et al., 2012 also reported the same. Among varieties Vandana produced significantly higher grain yield $\left(2988 \mathrm{~kg} \mathrm{ha}^{-1}\right)$ over other varieties consequently recorded higher net return and $\mathrm{B}: \mathrm{C}$ ratio similar to variety Anjali. Maximum yield loss of $29.86 \%$ occurred when no weeding was performed irrespective of rice varieties. Among different improved rive vatieties BVD-110, Vandana and Anjali recorded yield loss to the tune of 5.24, 7.01

\begin{tabular}{|c|c|c|c|c|c|c|c|c|c|c|c|c|c|}
\hline \multirow[t]{4}{*}{ Rice varieties } & \multicolumn{12}{|c|}{ Weed species } & \multirow{4}{*}{$\begin{array}{c}\begin{array}{c}\text { Alternan- } \\
\text { thera sessilis }\end{array} \\
75 \text { DAS }\end{array}$} \\
\hline & \multicolumn{3}{|c|}{ Cyperus rotundus } & \multicolumn{3}{|c|}{ Cynodon dactylon } & \multicolumn{3}{|c|}{ Bulbostilis burbata } & \multicolumn{3}{|c|}{ Ludwigia parviflora } & \\
\hline & 25 & 50 & 75 & 25 & 50 & 75 & 25 & 50 & 75 & 25 & 50 & 75 & \\
\hline & DAS & DAS & DAS & DAS & DAS & DAS & DAS & DAS & DAS & DAS & DAS & DAS & \\
\hline Vandana & 57.64 & 13.07 & 79.58 & 62.20 & 51.00 & 34.58 & 35.02 & 25.00 & 13.97 & 8.30 & 17.26 & 16.89 & 84.25 \\
\hline BVD 109 & 9.86 & 60.04 & 9.94 & 20.46 & 70.10 & 3.32 & 28.80 & 41.75 & 48.88 & 63.64 & 3.97 & 8.22 & 73.38 \\
\hline BVD 110 & 10.86 & 12.45 & 5.20 & 75.77 & 11.79 & 6.21 & 0.77 & 5.96 & 60.07 & 19.15 & 35.94 & 16.86 & 90.00 \\
\hline Anjali & 38.87 & 52.24 & 18.89 & 39.86 & 52.96 & 39.77 & 40.51 & 12.82 & 57.13 & 16.79 & 23.59 & 10.28 & 64.50 \\
\hline Kalinga 3 & 3.50 & 8.55 & 26.92 & 40.08 & 73.27 & 53.37 & 17.49 & 31.89 & 64.59 & 15.59 & 0.21 & 16.50 & 94.01 \\
\hline Brown gora 102 & 0.20 & 39.61 & 55.58 & 19.24 & 47.28 & 14.66 & 16.70 & 27.52 & 34.49 & 12.59 & 69.48 & 23.94 & 91.21 \\
\hline Annada & 28.37 & 30.19 & 21.59 & 40.60 & 30.00 & 49.87 & 0.06 & 20.31 & 49.81 & 20.56 & 50.50 & 42.91 & 29.50 \\
\hline Narendra 97 & 24.15 & 67.26 & 39.78 & 2.66 & 60.02 & 25.89 & 22.51 & 19.70 & 54.12 & 3.05 & 67.48 & 46.56 & 50.06 \\
\hline BirsaDhan 101 & 33.44 & 46.28 & 42.48 & 31.38 & 69.05 & 54.64 & 27.55 & 57.09 & 48.01 & 19.74 & 13.41 & 51.14 & 4.63 \\
\hline BirsaDhan 108 & 53.63 & 37.40 & 36.12 & 49.72 & 40.47 & 64.24 & 56.17 & 49.09 & 61.04 & 7.01 & 41.16 & 37.75 & 59.85 \\
\hline Tanrbhojna & 38.49 & 44.14 & 17.46 & 37.15 & 6.21 & 40.45 & 61.76 & 6.23 & 63.07 & 25.31 & 36.83 & 25.46 & 58.31 \\
\hline Asanleva & 0.10 & 42.98 & 59.70 & 29.95 & 7.62 & 71.31 & 5.72 & 24.49 & 44.23 & 30.00 & 21.84 & 50.78 & 56.34 \\
\hline Balibhojna & 16.89 & 31.23 & 17.85 & 42.22 & 7.07 & 37.20 & 19.53 & 28.45 & 64.27 & 45.96 & 4.69 & 21.32 & 75.47 \\
\hline $\begin{array}{l}\text { Mean relative } \\
\text { weed composi- } \\
\text { tion }(\%)\end{array}$ & 31.88 & 38.70 & 34.04 & 35.38 & 49.57 & 41.54 & 27.57 & 26.36 & 51.05 & 23.15 & 32.22 & 30.22 & 60.06 \\
\hline
\end{tabular}




\begin{tabular}{lcccccc}
\hline Rice varieties & \multicolumn{7}{c}{ Weed species } \\
\cline { 2 - 7 } & \multicolumn{7}{c}{ Other NL } & \multicolumn{3}{c}{ Other BL } \\
\cline { 2 - 7 } & 25 DAS & 50 DAS & 75 DAS & 25 DAS & 50 DAS & 75 DAS \\
\hline Vandana & 14.21 & 42.18 & 26.06 & 24.38 & 10.71 & 68.00 \\
BVD 109 & 19.46 & 33.69 & 16.44 & 75.97 & 7.96 & 36.55 \\
BVD 110 & 6.92 & 28.68 & 21.71 & 0.50 & 51.06 & 31.09 \\
Anjali & 58.57 & 21.49 & 29.14 & 35.67 & 3.32 & 21.68 \\
Kalinga 3 & 32.86 & 18.30 & 29.15 & 58.80 & 28.38 & 77.33 \\
Brown gora 102 & 79.34 & 13.94 & 34.08 & 76.36 & 13.12 & 11.14 \\
Annada & 48.25 & 26.32 & 34.45 & 52.46 & 71.06 & 5.49 \\
Narendra 97 & 32.72 & 48.50 & 15.21 & 58.99 & 30.39 & 13.62 \\
BirsaDhan 101 & 54.72 & 16.13 & 20.77 & 73.72 & 7.09 & 2.18 \\
BirsaDhan 108 & 15.96 & 5.25 & 11.58 & 87.47 & 49.78 & 7.56 \\
Tanrbhojna & 32.93 & 48.15 & 38.77 & 19.84 & 13.53 & 45.51 \\
Asanleva & 10.82 & 6.35 & 56.80 & 56.17 & 76.52 & 20.06 \\
Balibhojna & 75.08 & 4.41 & 27.19 & 93.42 & 28.79 & 67.02 \\
Mean relative weed & 40.06 & 26.55 & 27.53 & 55.76 & 32.40 & 34.27 \\
composition (\%) & & & & & & \\
\hline
\end{tabular}

Table 5: Influence of weeding and rice varieties on growth, yield and economics

\begin{tabular}{lcccccccccccc}
\hline Treatments & DA & DM & PH & ET & PL & GP & SW & YKH & SY & RYL & NR & B:C \\
\hline Weed control & & & & & & & & & & & & \\
Weedy check & 69.56 & 90.18 & 82.55 & 220.17 & 16.99 & 37 & 21.14 & 1655 & 2604 & 29.86 & 7303 & 1.67 \\
Weeding & 66.23 & 86.64 & 84.14 & 240.96 & 17.55 & 49 & 22.00 & 2212 & 3620 & 0.00 & 11384 & 1.82 \\
SEm \pm & 0.28 & 0.24 & 1.04 & 11.88 & 0.23 & 0.97 & 0.16 & 41.92 & 72.48 & 0.97 & 351 & 0.03 \\
CD ( $p=0.05)$ & 0.80 & 0.67 & NS & NS & NS & 2.74 & 0.45 & 118.66 & 205.15 & 2.74 & 992 & 0.08 \\
Variety & & & & & & & & & & & & \\
Vandana & 66.17 & 84.50 & 95.97 & 366.33 & 19.35 & 50 & 24.96 & 2988 & 5147 & 10.47 & 21834 & 2.77 \\
BVD109 & 64.67 & 85.17 & 92.67 & 266.67 & 18.12 & 48 & 23.57 & 2653 & 4572 & 7.01 & 18005 & 2.47 \\
BVD110 & 66.33 & 86.00 & 83.10 & 248.67 & 16.90 & 47 & 22.78 & 2638 & 4397 & 5.24 & 17539 & 2.44 \\
Anjali & 65.33 & 85.00 & 93.57 & 321.00 & 18.73 & 49 & 23.69 & 2794 & 4887 & 8.60 & 19766 & 2.61 \\
Kalinga-3 & 65.83 & 85.83 & 65.50 & 125.67 & 14.62 & 42 & 20.99 & 1152 & 1833 & 28.98 & 524 & 1.00 \\
BG102 & 66.50 & 85.67 & 72.53 & 182.33 & 15.63 & 44 & 21.49 & 1452 & 2341 & 24.30 & 3940 & 1.28 \\
Annada & 70.33 & 84.67 & 58.67 & 86.33 & 13.75 & 41 & 20.15 & 1013 & 1648 & 31.62 & -958 & 0.88 \\
Narendra97 & 73.83 & 86.50 & 80.77 & 239.67 & 16.85 & 46 & 22.24 & 2074 & 3295 & 20.33 & 10819 & 1.84 \\
BD101 & 62.17 & 84.33 & 70.90 & 158.33 & 15.50 & 43 & 21.18 & 1338 & 2130 & 22.97 & 2605 & 1.18 \\
BD108 & 63.33 & 86.50 & 79.68 & 235.00 & 16.37 & 45 & 21.82 & 1956 & 2925 & 19.13 & 9134 & 1.71 \\
Tanrbhajna & 70.50 & 97.50 & 107.77 & 227.60 & 20.50 & 35 & 17.08 & 1344 & 2288 & 6.02 & 2968 & 1.25 \\
Asanleva & 73.67 & 96.33 & 93.67 & 273.80 & 17.93 & 37 & 22.23 & 2127 & 2685 & 4.59 & 10025 & 1.83 \\
Balibhojna & 74.00 & 101.33 & 88.73 & 266.00 & 20.23 & 34 & 18.21 & 1625 & 2314 & 4.83 & 5264 & 1.44 \\
SEm \pm & 0.77 & 0.65 & 2.85 & 32.52 & 0.62 & 3 & 0.43 & 114.81 & 198.50 & 2.65 & 960 & 0.08 \\
CD ( $p=0.05)$ & 2.19 & 1.83 & 8.06 & 92.05 & 1.76 & 8 & 1.22 & 324.97 & 561.84 & 7.51 & 2717 & 0.22 \\
Interaction & & & & & & & & & & & & \\
\hline & & & & & & & & & & & $C 0 n t n u e .$.
\end{tabular}




\begin{tabular}{lcccccccccccc}
\hline Treatments & DA & DM & PH & ET & PL & GP & SW & YKH & SY & RYL & NR & B:C \\
\hline SEm \pm & 1.09 & 0.91 & 4.03 & 45.99 & 0.88 & 3.75 & 0.61 & 162.37 & 280.72 & 3.75 & 1358 & 0.11 \\
CD $(p=0.05)$ & NS & NS & NS & NS & NS & NS & NS & 459.58 & 794.56 & 10.63 & 3842 & 0.31 \\
\hline
\end{tabular}

DA: Days to anthesis; DM: Days to maturity; PH: Plant ht (cm); ET: Effective tillers/m²; PL: Panicle length; GP: Grains panicle ${ }^{-1}$; SW: 1000 seed wt; YKH: Yield kg ha-1; SY: Straw yield kg ha-1 ${ }^{-1}$ RYL: Relative yield loss (\%); NR: Net return (₹ ha-1); B:C ratio was calculated on the basis of gross return; Price of paddy- ₹ $8 / \mathrm{kg}$

and $8.6 \%$ respectively. However, traditional varieties Asanleva, Balibhojna and Tanrbhojna recorded yield loss to the extent of $4.59,4.83$ and $6.02 \%$ indicating weed suppressing ability compared to others.

\section{Conclusion}

Two hand weeding at 20 and 40 days after sowing suppressed weeds effectively producing higher yield. Among different varieties, Vandana being more tolerant to weed pressure than other varieties could be recommended to farmers in the study areas with similar environmental conditions as a first choice variety in relation to the other varieties studied.

\section{References}

Anwar, P. Juraimi, A.S. Man, A., Puteh, A., Selamat, A., Begum, M., 2010. Weed suppressive ability of rice (Oryza sativa L.) germplasm under aerobic soil conditions," Australian Journal of Crop Science 4(9), 706-717.

Evans, S.P., Knezevic, S.Z., Lindquist, J.L., Shapiro, C.A., 2003. Influence of nitrogen and duration of weed interference on corn growth and development. Weed Science 51(4), 546-556.

Fischer, A.J., Ramirez, H.V., Gibson, K.D., Pinheiro, B., Da Silveira, 2001. Competitiveness of semi dwarf upland rice cultivars againstpalisadegrass (Brachiaria brizantha) and signalgrass (B. decumbens)," Agronomy Journal 93(5), 967-973

Harding, S.S., Jalloh, A.B., 2013. Evaluation of the relative weed competitiveness of some lowland rice varieties in sierra leone. American Journal of Experimental Agriculture 3(2), 252-261.
Jadhav, A.S., Pawar, S.U., 2013. Time of sowing and influence of weed control methods on yield of direct seeded rice. Journal of Agriculture Research Technology 38(3), 466-469.

Kumar, J., Singh, D., Singh, B., Singh, R., Pawar, S., Gupta K., 2012. sowing time and weed management practices to enhance yield of direct seeded rice. Indian Journal of Weed Science 44(4) 207-209.

Labrada, R., 2003. The need for improved weed management in rice; in: Proceedings of the $20^{\text {th }}$ Session of the International Rice commission Bangkok, Thailand, 23-26, July.

Mishra, J.S., singh, V.P., 2008. Integrated weed management in dry-seeded irrigated rice (Oryza sativa). Indian Journal of Agronomy 52(4), 299-300.

Shetty, S.V.R., Rao, A.N., 1979. weed management studies in sorghum/pigeonpea and pearl millet/groundnut intercropping systems some observations In: Proceedings of International Workshop on Intercropping, 10-13, January, ICRISAT, Hyderabad, India.

Singh, M., Sairam, C.V., Hanji, M.B., Prabhukumar, S., Nandkishor, 2012. Crop weed competition and weed management studies in direct seeded rice (Oryza sativa). Indian Journal of Agronomy 57(1), 38-42.

Zhao, D.L., Atlin, G.N., Bastiaans, L., Spiertz, J.H.J., 2006. Comparing rice germplasm groups for growth, grain yield and weed-suppressive ability under aerobic soil conditions. Weed Research 46(6), 444-452. 